\title{
Mohs micrographic surgery in Singapore: A long-term follow-up review
}

\begin{abstract}
Dear Editor,
Mohs micrographic surgery (MMS) has become the treatment of choice for high-risk non-melanoma skin cancers (NMSC) worldwide. ${ }^{1}$ Unlike wide local excision, MMS enables precise microscopic removal of tumour foci while allowing maximal tissue preservation, thereby maintaining cosmesis and anatomical function. ${ }^{1}$ The MMS service in Singapore started in 2008, and we present our experience with MMS at the National Skin Centre, Singapore.
\end{abstract}

A retrospective review of patients who underwent MMS performed by 4 different Mohs surgeons over a 4-year period from January 2010 to December 2013 was conducted. Information collected included demographic data, disease characteristics and treatment details. Specific treatment outcomes assessed were post-surgical complication rate and 5-year recurrence rate. This study was approved by the institutional ethics review board.

A total of 481 tumours were removed from 426 patients by MMS during the study period. Patients' median age was 67.0 years (range $29-97$ years). There were $54.7 \%$ (233/426) men and 45.3\% (193/426) women. Majority of the patients were Chinese at $75.8 \%(323 / 426)$, followed by Caucasians at $15.3 \%(65 / 426)$, Malays at $1.9 \%(9 / 426)$ and Indians at $0.8 \%(4 / 426)$. Among them, 28 patients $(6.6 \%)$ had $>1$ tumour removed with MMS in a single sitting.

There were $462(96.1 \%)$ primary tumours and 19 (3.9\%) recurrent tumours. All recurrent tumours were basal cell carcinomas (BCCs). All recurrent BCCs had at least 1 other high-risk clinical and/or histological feature as defined by the US National Comprehensive Cancer Network Guidelines (2017). ${ }^{2}$ Ten were located on high-risk anatomical sites (nose $[\mathrm{n}=8]$, ears $[\mathrm{n}=2]$ and periocular $[\mathrm{n}=1])$ and 8 had aggressive histological subtypes (6 infiltrative BCCs and 2 micronodular $\mathrm{BCCs}$ ). Initial treatment modalities utilised for the primary tumours of recurrent BCCs included MMS $(n=5)$, wide local excision $(n=11)$, liquid nitrogen $(n=2)$ and topical imiquimod $(\mathrm{n}=1)$.

The most common malignancy was BCC at $91.9 \%$ (442/481), followed by squamous cell carcinoma (SCC) at $7.5 \%(36 / 481)$, and 1 case each of atypical fibroxanthoma, sebaceous carcinoma and extramammary Paget's disease. The predominant site of involvement was the head and neck region at $98.3 \%$ (473/481), followed by the trunk at $1.2 \%(6 / 481)$, genitalia at $0.2 \%(1 / 481)$ and digits at $0.2 \%(1 / 481)$.
Standard MMS with frozen sections was performed for most tumours $(95.4 \%, 459 / 481)$. Twenty-two tumours $(4.6 \%)$ required the use of paraffin-embedded sections and/ or "slow Mohs" procedure. The mean pre- and postoperative sizes were $153.7 \pm 222.7 \mathrm{~mm}^{2}$ and $345.2 \pm 452.2 \mathrm{~mm}^{2}$, respectively (median $=90$ and 208, respectively).

Complete removal of tumours required a mean of 1.6 surgical stages. There were 17 tumours (3.5\%) comprising 13 BCCs, 3 SCCs and 1 sebaceous carcinoma, which required at least 4 surgical stages for removal, and all these tumours had high-risk features ${ }^{2}-11$ BCCs were located on the nose, 4 BCCs were recurrent tumours, 3 BCCs had aggressive histological features (2 micronodular BCCs and 1 basosquamous BCC), 2 SCCs had perineural invasion and $1 \mathrm{SCC}$ had invasion down to the bone.

Most surgical defects $(88.5 \%, 426 / 481)$ were closed by the surgeon performing the procedure. Linear direct closure was possible in $221(45.9 \%)$ cases. There were 109 $(22.7 \%)$ cases that required a variety of random patterned flaps, including $43(8.9 \%)$ rotation flaps, 42 (8.7\%) advancement flaps, 17 (3.5\%) transposition flaps and 7 $(1.5 \%)$ island pedicle flaps. There were 48 cases $(10 \%)$ that required full-thickness skin grafts (FTSG). A combination of closure techniques was employed for 37 cases $(7.7 \%)$. Eleven patients $(2.3 \%)$ had secondary intention healing, one of whom had the defect reduced in size by purse string closure; 55 (11.2\%) patients required assistance from oculoplastic/plastic surgeons for defect closure; and 1 patient $(0.2 \%)$ required reconstruction by a hand surgeon after MMS for a left middle finger SCC. Fig. 1 illustrates a patient with MMS and in-house reconstruction performed at our centre.

There were $48(10 \%)$ patients who were lost to follow-up postoperatively and excluded from treatment outcome analysis. Immediate post-surgical complications affected 15 patients $(3.1 \%)$, of whom $11(2.3 \%)$ had superficial tip necrosis of surgical flaps/grafts that did not affect their final surgical outcomes; $2(0.4 \%)$ had mild wound dehiscence; $1(0.2 \%)$ had minor surgical site bleeding; and $1(0.2 \%)$ had minor wound infection.

The median follow-up duration after MMS was 35.8 months (range 1.1-101.8). Recurrence of NMSCs occurred in 3 patients $(0.6 \%)$ and were detected during the 3-6 monthly follow-up visits after MMS. Two out of 3 recurrent tumours were nodular BCCs at high-risk areas. One BCC recurred over the left ear, with the previous defect after MMS left to heal by secondary intention. The other recurrent $\mathrm{BCC}$ was located on the nasal tip adjacent to the patient's FTSG scar. Both 


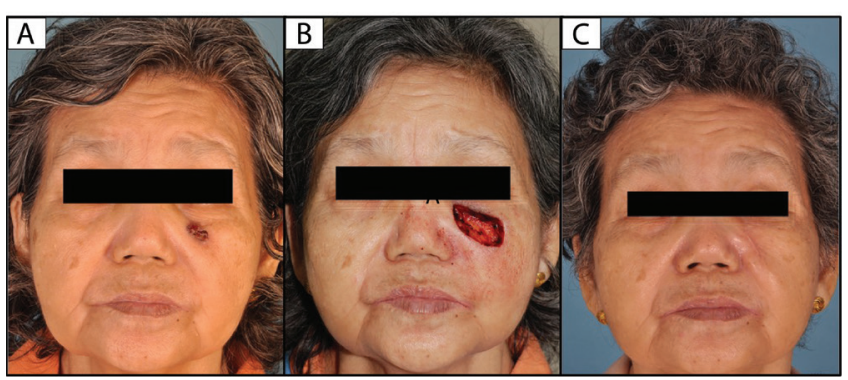

Fig. 1. (A) 19x10mm nodular basal cell carcinoma on the left cheek. (B) 26x20mm defect after Mohs micrographic surgery (MMS). (C) Reconstruction with a unilateral cheek advancement flap (appearance 1 year after MMS).

recurrent BCCs were treated with repeat MMS. The remaining recurrent tumour was a high-risk moderately differentiated SCC due to site (cheek) and size (13mm). It was treated with wide local excision and adjuvant radiotherapy. The median time from MMS to recurrence was 24.8 months (range 1.5-53.3).

To the best of our knowledge, this is one of the first studies evaluating the role of MMS in Singapore. While most tumours in our series were treated with standard MMS with frozen sections, MMS with formalin-fixed, paraffin-embedded sections was employed for rarer cutaneous tumours such as atypical fibroxanthoma, sebaceous carcinoma and extramammary Paget's disease. Other NMSCs that may be treated with this modified MMS include microcystic adnexal carcinoma, ${ }^{3}$ dermatofibrosarcoma protuberans, ${ }^{4}$ lentigo maligna ${ }^{5}$ and melanoma. ${ }^{6}$ These tumours often have insidious single cell spread that is easily missed on frozen sections, and using paraffin sections minimises the risk of falsenegative interpretation resulting from fat dropout and freeze artefacts. ${ }^{7}$ For SCCs and BCCs in our series that had aggressive histological subtypes demonstrating single cell invasion and/or have perineural involvement $(n=22)$, it is also our practice to send the final sections of these specimens for paraffin sections. While this entailed delayed closure, the additional margin check prior to defect closure provides greater accuracy which may minimise recurrence risk.

We were able to reconstruct the defects in majority of our patients. With smaller defects achieved through MMS compared to wide local excision, linear direct closure was possible in almost half of the cases. For larger defects that crossed cosmetic units, a combination of closure techniques that allowed closure with minimal skin movement were utilised, thereby avoiding complex reconstructive procedures and reducing patients' postoperative morbidity.

The main limitation of our study is its retrospective nature. Furthermore, $10 \%$ of patients were lost to follow-up, which affected assessment of treatment outcomes. This was not surprising given the sizeable proportion of expatriate and elderly patients (median age 67 years) in our cohort, some of whom might have returned to their countries postoperatively or not return for follow-up if they were well.

In conclusion, our results demonstrate the effectiveness and safety of MMS for high-risk NMSC with an overall low 5-year recurrence rate $(0.6 \%)$ and complication rate (3.1\%). This was comparable to the data reported by Rowe et al., who observed a recurrence rate of $1 \%$ and $3 \%$ for primary $\mathrm{BCC}$ and primary SCC, respectively ${ }^{8-10}$ after MMS. "Slow Mohs" has also been performed for rarer NMSC with good outcomes.

\section{REFERENCES}

1. Cumberland L, Dana A, Liegeois N. Mohs Micrographic Surgery for the Management of Nonmelanoma Skin Cancers. Facial Plast Surg Clin North Am 2009;17:325-35.

2. National Comprehensive Cancer Network. NCCN Guidelines. Basal Cell Skin Cancer. Available at: https://www.ncen.org/ guidelines/guidelines-detail?category $=1 \& i d=1416$. Accessed on 18 October 2021

3. Diamantis SA, Marks VJ. Mohs micrographic surgery in the treatment of microcystic adnexal carcinoma. Dermatol Clin 2011; 29:185-90, viii.

4. Zhou X, Sun D, Liu Y, et al. Dermatofibrosarcoma protuberans: our 10-year experience on 80 patients. J Dermatolog Treat 2020; 31:554-8.

5. Cohen LM, McCall MW, Zax RH. Mohs micrographic surgery for lentigo maligna and lentigo maligna melanoma. A follow-up study. Dermatol Surg 1998;24:673-7.

6. Ellison PM, Zitelli JA, Brodland DG. Mohs micrographic surgery for melanoma: A prospective multicenter study. J Am Acad Dermatol 2019;81:767-74

7. Orchard GE, Shams M. Dermatofibrosarcoma protuberans: dealing with slow Mohs procedures employing formalin-fixed, paraffin wax-embedded tissue in a busy diagnostic laboratory. Br J Biomed Sci 2012;69:56-61.

8. Rowe DE, Carroll RJ, Day CL Jr. Long-term recurrence rates in previously untreated (primary) basal cell carcinoma: implications for patient follow-up. J Dermatol Surg Oncol 1989;15:315-28.

9. Rowe DE, Carroll RJ, Day CL Jr. Mohs surgery is the treatment of choice for recurrent (previously treated) basal cell carcinoma. J Dermatol Surg Oncol 1989;15:424-31.

10. Rowe DE, Carroll RJ, Day CL Jr. Prognostic factors for local recurrence, metastasis, and survival rates in squamous cell carcinoma of the skin, ear, and lip. Implications for treatment modality selection. J Am Acad Dermatol 1992;26:976-90.

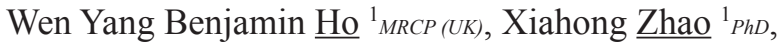
Wee Ping Melissa $\underline{\operatorname{Tan}}^{1}{ }^{1}$ MRCP (UK)

${ }^{1}$ National Skin Centre, Singapore

Correspondence: Dr Wen Yang Benjamin Ho, National Skin Centre, 1 Mandalay Road, Singapore 308205.

Email: benjamin.ho@mohh.com.sg 